\title{
Measurement of the LOFAR-HBA beam patterns using an unmanned aerial vehicle in the near field
}

\author{
Giuseppe Virone $\odot,{ }^{\mathbf{a}}$ Fabio Paonessa $\odot,{ }^{\mathbf{a}}$ Lorenzo Ciorba $\odot,{ }^{\mathrm{a}} *$ \\ Stefania Matteoli $\odot,{ }^{\text {a }}$ Pietro Bolli $\odot,{ }^{b}$ Stefan J. Wijnholds $\odot,{ }^{c}$ and \\ Giuseppe Addamo ${ }^{\text {a }}$

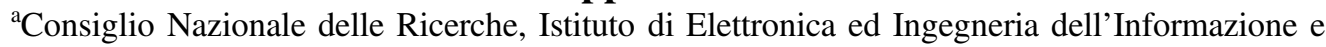 \\ delle Telecomunicazioni, Turin, Italy \\ ${ }^{\mathrm{b}}$ Istituto Nazionale di Astrofisica, Osservatorio Astronomico di Arcetri, Florence, Italy \\ ${ }^{\mathrm{c}}$ Netherlands Institute for Radio Astronomy, Dwingeloo, Netherlands
}

\begin{abstract}
An unmanned aerial vehicle (UAV) is exploited to characterize in situ the high-band antennas (HBAs) of the low-frequency array (LOFAR) CS302 station located in Exloo, The Netherlands. The size of an HBA array is about $30 \mathrm{~m}$. The Fraunhofer distance (a few kilometers) is not reachable in the frequency band (120 to $240 \mathrm{MHz})$ within the flight regulation limits. Therefore, far-field patterns cannot be directly measured. The UAV, equipped with an radio frequency synthesizer and a dipole antenna, flies in the near-field region of the considered array. Measurement of three different frequencies $(124,150$, and $180 \mathrm{MHz})$ is efficiently made during the same UAV flight. The near-field focusing method is exploited to validate the far-field pattern of the array under test within an angular range around the beam axis. Such a technique avoids both the time consuming $\lambda / 2$ sampling of the aperture field and the further application of computationally heavy near-field to far-field transformations. The array beam is well reconstructed in the main lobe and first sidelobes within a 2D scan plane sampled with a radial raster. A further postprocessing technique is proposed and validated on a subarray of HBAs. It suggests efficient ways for the future characterization of regular aperture arrays for SKA-MID Phase 2. $\odot$ The Authors. Published by SPIE under a Creative Commons Attribution 4.0 International License. Distribution or reproduction of this work in whole or in part requires full attribution of the original publication, including its DOI. [DOI: 10.1117/1.JATIS.8.1.011005]
\end{abstract}

Keywords: antenna measurements; unmanned aerial vehicle; near-field focusing; very high frequency band; large arrays; hybrid beamforming.

Paper 21098SS received Aug. 23, 2021; accepted for publication Nov. 1, 2021; published online Nov. 23, 2021.

\section{Introduction}

The low-frequency array (LOFAR) $)^{1}$ is a radio telescope composed of 52 stations located in Europe. Each station is composed of two subarrays, one with low-band antennas (LBAs) and one with high-band antennas (HBAs). Their operating frequency ranges are 10 to $90 \mathrm{MHz}$ and 120 to $240 \mathrm{MHz}$, respectively. LBAs are arranged in a random configuration, whereas HBAs are placed in a regular lattice.

LOFAR is a pathfinder for the international square kilometer array (SKA). The SKA will become the biggest and most sensitive radio telescope in the world. Aperture arrays are envisioned for both SKA1-LOW ( 50 to $350 \mathrm{MHz}$, random configuration) ${ }^{2,3}$ and possibly SKA1-Mid phase 2 (400 to $1.45 \mathrm{GHz}$, regular configuration).$^{4-6}$ Dishes will be adopted for higher frequencies up to $14 \mathrm{GHz}$.

All these powerful radio telescopes need to be validated and accurately calibrated. Of course, testing these large arrays is not an easy task due to their large size and the low operating frequencies. A few approaches have been proposed exploiting measured data in far or quasi-far-field condition. For example, a holographic technique has been applied to the engineering development array 2 of SKA1-LOW ${ }^{7}$ and $\mathrm{LOFAR}^{8}$ to retrieve aperture fields. Other tests on LOFAR have

*Address all correspondence to Lorenzo Ciorba, lorenzo.ciorba@ieiit.cnr.it 


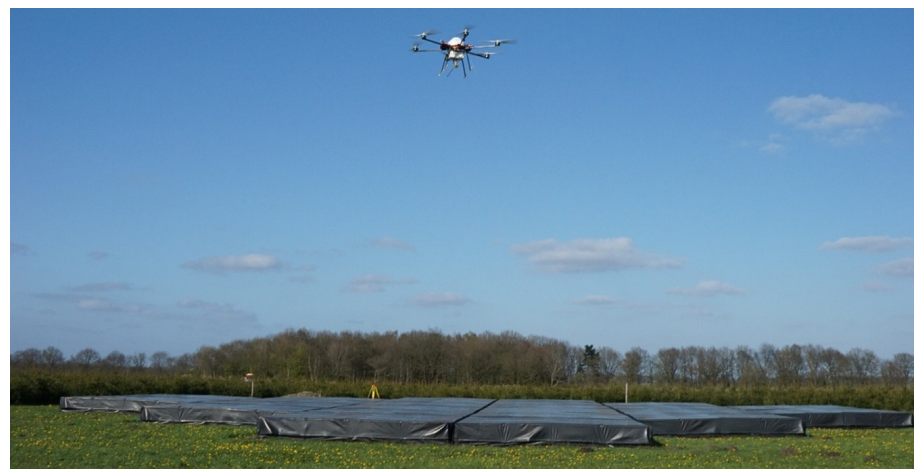

Fig. 1 The UAV after take-off is reaching the first waypoint to perform the required scan path over the LOFAR Eastern HBA array of the CS302 station. The array dimension is about $30 \mathrm{~m}$.

been performed using astronomical calibration sources ${ }^{9}$ and radio frequency (RF) sources mounted on cranes. ${ }^{10}$

More recently, thanks to the technological development of unmanned aerial vehicles (UAVs), flying test sources have been developed to test aperture arrays even at the element level ${ }^{11-16}$ with a high signal-to-noise ratio and a huge scan flexibility. Due to the large electrical size of the aperture arrays, the Fraunhofer distance cannot always be reached. Therefore, validation of LOFAR LBA array has been performed comparing measurement and simulation in the near field. ${ }^{17,18}$ All previous papers featured sparse arrays with particular emphasis on the random configuration. ${ }^{19}$ In this work, the UAV-based measurement strategy is extended to the LOFAR HBA (Fig. 1), which is a large regular array. Near-field focusing ${ }^{20,21}$ is adopted and its validity is assessed by comparison to the far-field simulated data (Sec. 2). This procedure has been applied on a two-dimensional scan path to provide a more complete characterization (Sec. 3). A first attempt to develop a far-field reconstruction strategy (to overcome the artifacts of the near-field focusing) and the corresponding definition of efficient near-field scan strategies for regular arrays such as the aperture arrays for SKA-MID Phase 2 is presented in Sec. 4.

To summarize, the novelty aspects of this paper are as follows:

(1) The verification of all tiles within a single flight over a LOFAR-HBA substation.

(2) The application of near-field focusing to an array of tiles pointed in the same far-field direction to provide an end-to-end verification of the system from the antennas to the digitized data.

(3) The usage of radial raster scans to represent the beam pattern in the $u-v$ plane with a limited set of linear scans, which is an efficient choice in view of the limited UAV flight duration compared to Cartesian rasters. ${ }^{11}$

(4) The definition of a far-field reconstruction strategy to partially overcome the artifacts of the near-field focusing and its validation on a subarray of the HBA substation.

\subsection{UAV-Mounted Test Source and the HBA}

The UAV is visible in Fig. 1. It is equipped with a differential global navigation satellite system (GNSS) receiver for accurate positioning within a few centimeters, a dipole antenna, and an RF synthesizer. ${ }^{13}$ Three higher-order harmonics of the RF synthesizer (i.e., it was operating as a comb generator) have been used during the same flight. In this way, three different frequencies (i.e., 124, 150, and $180 \mathrm{MHz}$ ) have been measured simultaneously. This procedure is applicable when the receiver acquisition system can simultaneously record many frequency channels to drastically reduce the flight time of the UAV.

Figure 1 also shows the Eastern HBA array of the CS302 LOFAR station located in Exloo. The detailed geometry of this LOFAR HBA subarray is shown in Fig. 2. It is composed of 24 square tiles (red numbers from 0 to 23) with a size of about $5 \times 5 \mathrm{~m}^{2}$. Each tile is composed of a regular distribution of $4 \times 4$ dual-polarized elements, i.e., thin crossed-bowtie dipoles suspended over a ground plane by means of a polystyrene support structure. The dipole length is about $0.7 \mathrm{~m}$. Within each tile, the element spacing is $1.25 \mathrm{~m} .{ }^{1}$ The distance between tile centers is 


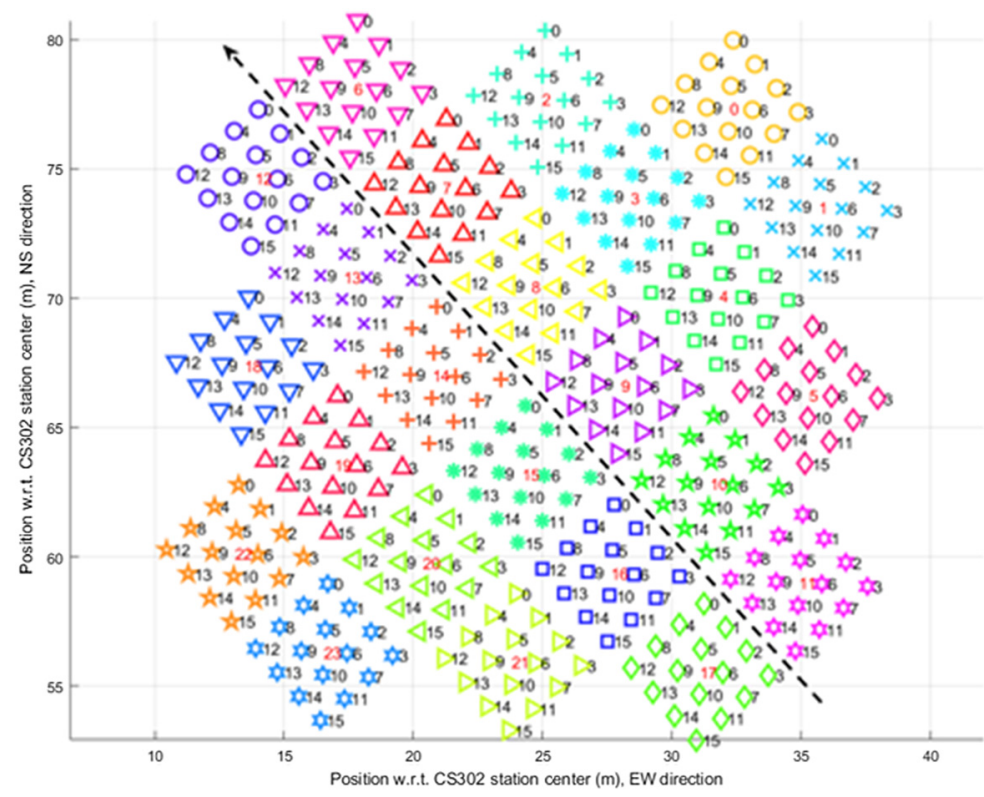

Fig. 2 Element positions in LOFAR HBA subarray. Red and black numbers refer to tile number (0-23) and element number (0-15) inside the single tile, respectively. The black dashed curve shows an example of a UAV path (its projection to the ground) oriented along the north-west direction.

$5.15 \mathrm{~m}$, therefore, the spacing between elements of adjacent tiles is slightly larger $(1.4 \mathrm{~m})$ than within the tile. Nevertheless, the overall distribution can be considered as very close to a uniform regular array. Through the analog beam forming, each tile can be pointed within a field of view of $60 \mathrm{deg}$ around zenith. Furthermore, signals from all tiles can be summed together by digital beam forming.

The two element polarizations are oriented along the north-west and north-east directions. For the considered CS302 station, the orientation of the regular array distribution is $48 \mathrm{deg}$ from north (see Fig. 2). Hence, there is a 3-deg rotation between element polarization directions and array lattice principal directions. Nevertheless, the labels north-west and north-east will be still adopted in this paper for both polarization and array principal (periodicity) directions for the sake of simplicity.

\section{Beam Patterns Using Near-Field Focusing}

Several flights with linear trajectories and constant height have been performed. A sketch of a UAV path projected to the ground and oriented north-west is shown in Fig. 2 with the blackdashed line. This section presents the results obtained for a flight where both the UAV speed vector and the onboard dipole are oriented north-west. This corresponds to an $E$-plane scan of the array elements oriented north-west. The UAV flight duration to perform this single linear path was $\sim 1$ min (flight speed was about $3 \mathrm{~m} / \mathrm{s}$ ), whereas 2 to $3 \mathrm{~min}$ are necessary for take-off and landing. The flight height has been maintained at $140 \mathrm{~m}$ due to regulation limitations. This already satisfies the far-field condition for each tile (Fraunhofer distance is $60 \mathrm{~m}$ at $180 \mathrm{MHz}$ ) but is not enough for the full array (about $1.1 \mathrm{~km}$ at $180 \mathrm{MHz}$ ). This fact is confirmed in Fig. 3, where the measured tile beams ( $E$-plane, $124 \mathrm{MHz}$ ) are shown for the tiles closest to the projection of the UAV path (dashed black line in Fig. 2), i.e., tiles 6-11 and 12-17. The effect of UAV pattern and path loss has been removed as in Ref. 22. All beams are reported with respect to the curvilinear abscissa on the UAV path. The origin of the curvilinear abscissa is set where the UAV path projection is closest to the center of the HBA array. The analog beam formers were programmed to point the tile beams at zenith. However, because of the low altitude of the UAV, the maxima of the tile beams occur at different values of the curvilinear abscissa. In particular, the maxima of tiles 11 and 17 occur at about $-13 \mathrm{~m}$, whereas the maxima of tiles 6 and 12 occur at $+13 \mathrm{~m}$. This is consistent with the distance between tile centers of about $26 \mathrm{~m}$. The successful 


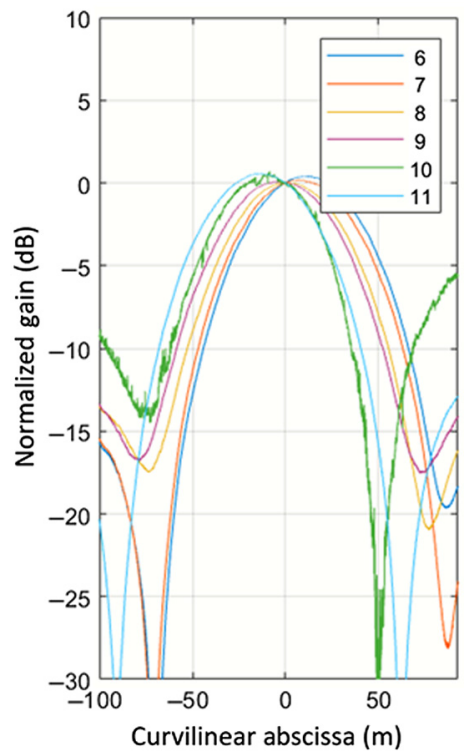

(a)

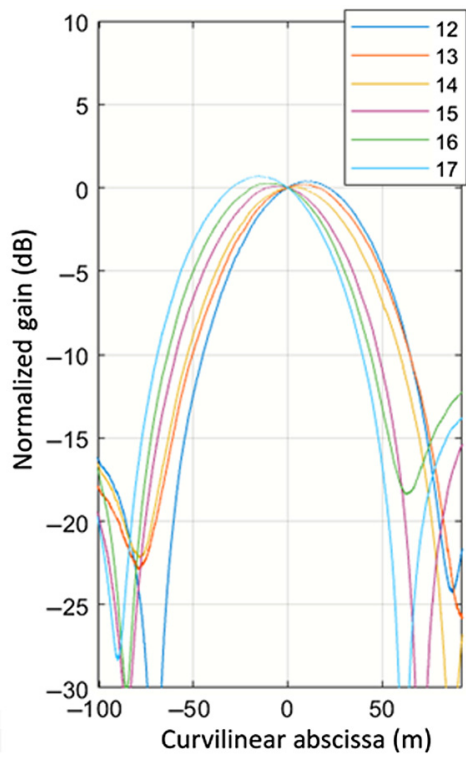

(b)

Fig. 3 Normalized E-plane radiation pattern for tiles (a) 6-11 and (b) 12-17 at $124 \mathrm{MHz}$.

comparison with simulations at tile level was already reported in Ref. 23. In this work, the tile beams are instead used to estimate the full array pattern by means of a near-field focusing method. ${ }^{20}$ However, it should be mentioned that, differently from Ref. 17, the presence of analog beam-formers at the tile level prevents the application of the required parabolic phase shifts across the array aperture, i.e., to each array element. Such near-field focusing can only be applied on the tile signals. In other words, the tile beams are pointed to zenith (far field), whereas the array of tiles will be focused in near field. All the tile beams in Fig. 3 are normalized in magnitude and phase at the origin of the curvilinear abscissa to produce the near-field focusing for the array of tiles. The parabolic phase shifts for the various tiles are automatically produced by the different electrical distances with respect to the UAV-mounted source placed in the near field.

Normalization constants in magnitude are shown in Fig. 4. It is apparent that tile number 10 requires a larger normalization constant with respect to the other tiles to achieve proper equalization. From Fig. 3, it can be also noted that its signal is noisier and the sidelobes are

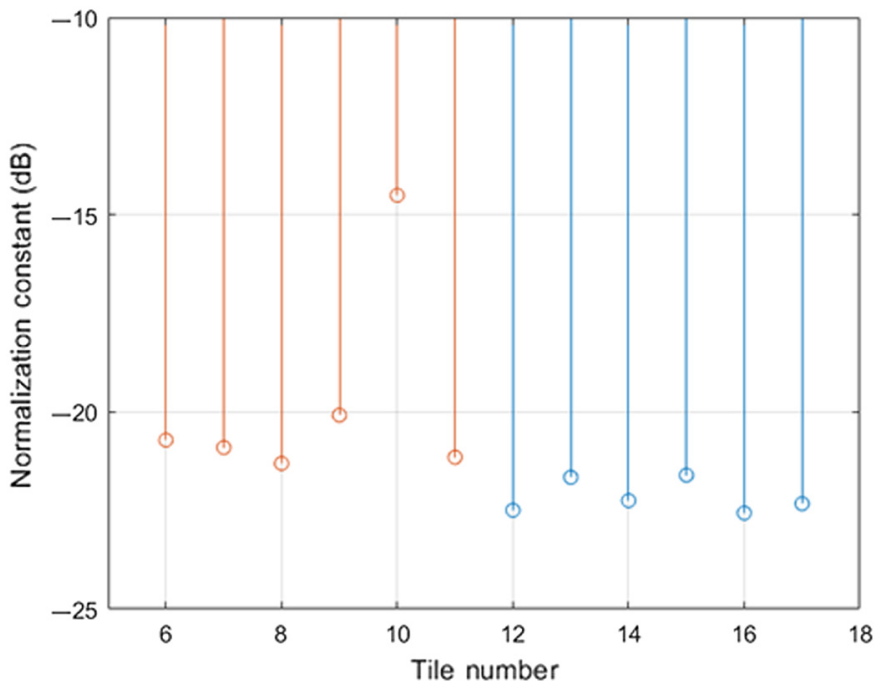

Fig. 4 Normalization constants (dB) for tiles 6-17 at $124 \mathrm{MHz}$. 


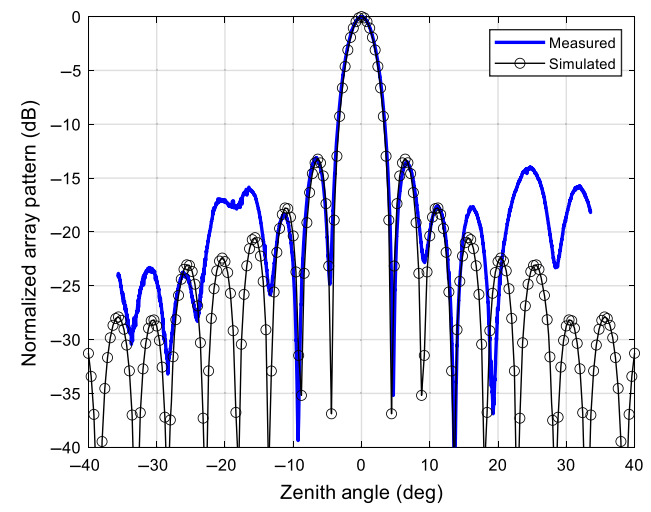

(a)

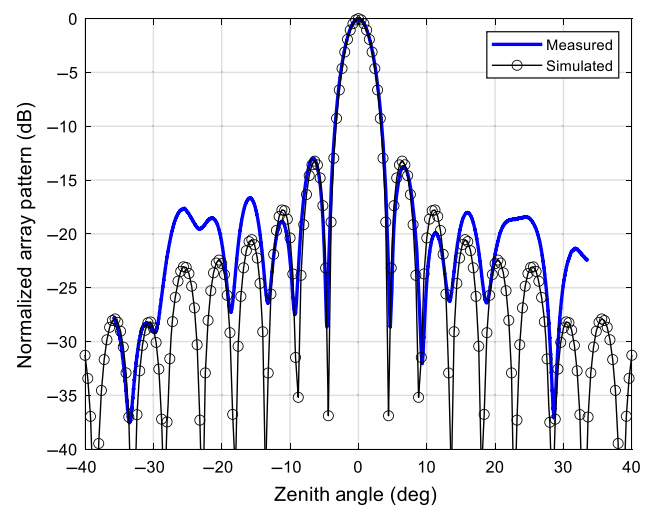

(b)

Fig. 5 Far-field pattern (black line) and focused near field (blue line) of tiles (a) 6-11 and (b) 12-17 at $124 \mathrm{MHz}$.

higher with respect to all the other tiles. This can be explained with a fault in the analog beam former of tile 10 .

The equalized (magnitude and phase) signals from the two tile rows 6-11 and 12-17 have been summed together to obtain the radiation patterns at $124 \mathrm{MHz}$ that are shown in Fig. 5 (blue line) as a function of the zenith angle. The zenith angle has been computed using the curvilinear abscissa and the UAV height. A far-field simulation obtained using a combination of WIPL-D and array factor is also shown in Fig. 5 (black solid line with circular markers). WIPL-D has been used on a subarray of $3 \times 3$ tiles, i.e., $12 \times 12$ dipoles. This configuration requires neither significant computational effort nor specific acceleration methods. However, it allows one to estimate the effect of mutual coupling on the tile beam. Differences between the nine simulated tile beams in the $3 \times 3$ tile array have been found to be negligible with respect to the measured discrepancies. ${ }^{23}$ For this reason, an array factor approach has been adopted using the simulated tile beam (central tile within the $3 \times 3$ array) as element pattern. The agreement is satisfactory within \pm 15 deg from zenith. This is consistent with the near-field focusing method, which guarantees a good agreement between far-field and near-field focused beams only in the proximity of the beam axis. ${ }^{20}$ The level of first sidelobes $(-13 \mathrm{~dB})$ is consistent with the uniform amplitude excitation. The pattern of the array of tiles 6-11 shows larger discrepancies with respect to simulation because of the faulty tile 10. Almost the same level of agreement has been obtained at 150 and $180 \mathrm{MHz}$ (see Fig. 6, only the array of tiles 12-17 has been reported for brevity). As expected, the angular region with good agreement becomes narrower at higher frequencies ( $\pm 10 \mathrm{deg}$ from zenith) because the Fraunhofer condition increases with frequency and all frequencies were measured during the same flight and, hence, at the same height.

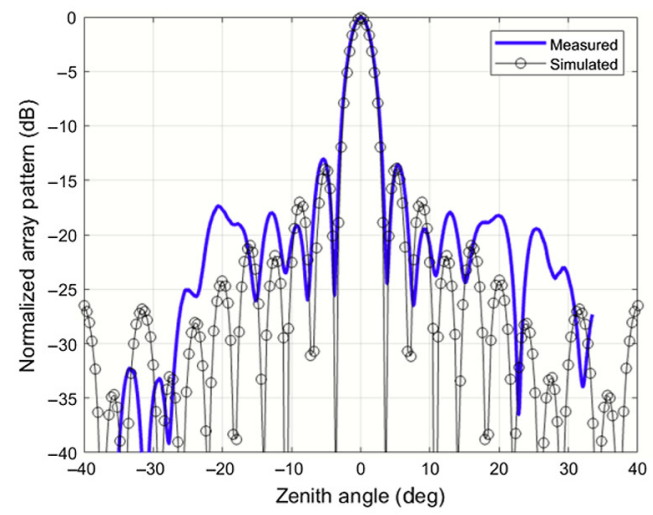

(a)

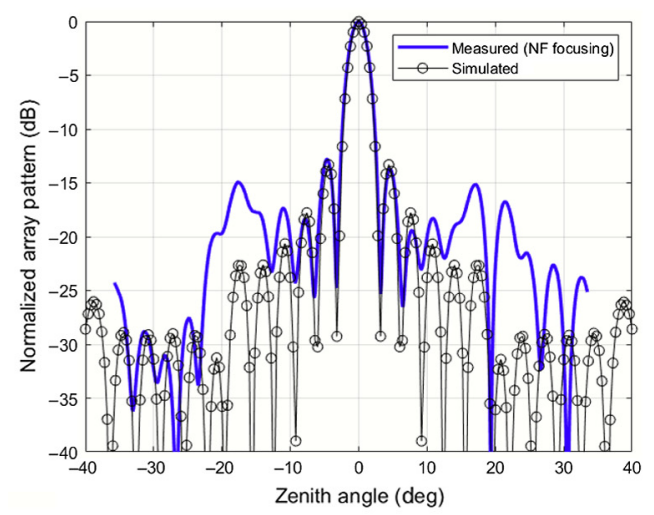

(b)

Fig. 6 Far-field pattern (black line) and focused near field (blue line) of tiles $12-17$ at (a) $150 \mathrm{MHz}$ and (b) $180 \mathrm{MHz}$. 


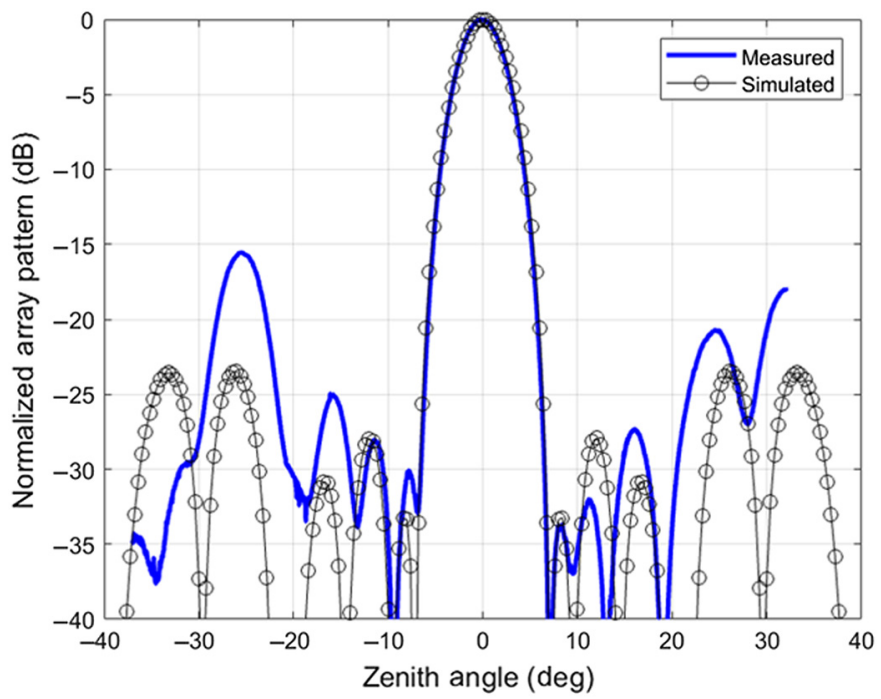

Fig. 7 Far-field pattern (black line) and focused near field (blue line) of tiles 1-24 (full HBA subarray) at $124 \mathrm{MHz}$.

The same near-field focusing procedure has been applied to the full HBA array in Fig. 2 . No further faulty tile has been found besides number 10. (It should be noted that all the tiles in Fig. 2 have been verified in such a single flight.) Figures 7-9 show the measured (near-field focused) and the simulated (far field) radiation patterns at 124, 150, and $180 \mathrm{MHz}$. The angular region showing good agreement is again ranging from \pm 15 deg to \pm 10 deg at lower and higher frequencies, respectively.

The full-half power beamwidths are $5.2 \mathrm{deg}, 3.9 \mathrm{deg}$, and $3.4 \mathrm{deg}$ at 124, 150, and $180 \mathrm{MHz}$, respectively. The low level of the first sidelobes is due to the array geometry. It should be noted that all the tiles in Fig. 2 contribute to the array pattern. The number of tiles along the direction that is orthogonal to the UAV scan, i.e., the number of tiles along north-east direction is six in the array center and two at its edges. As far as the north-west cut reported in Figs. 7-9 is concerned, this is equivalent (in the far field) to a linear array with edge tapering, which in turn explains the low sidelobes. This fact can be easily demonstrated by computing the array factor along the north-west cut.

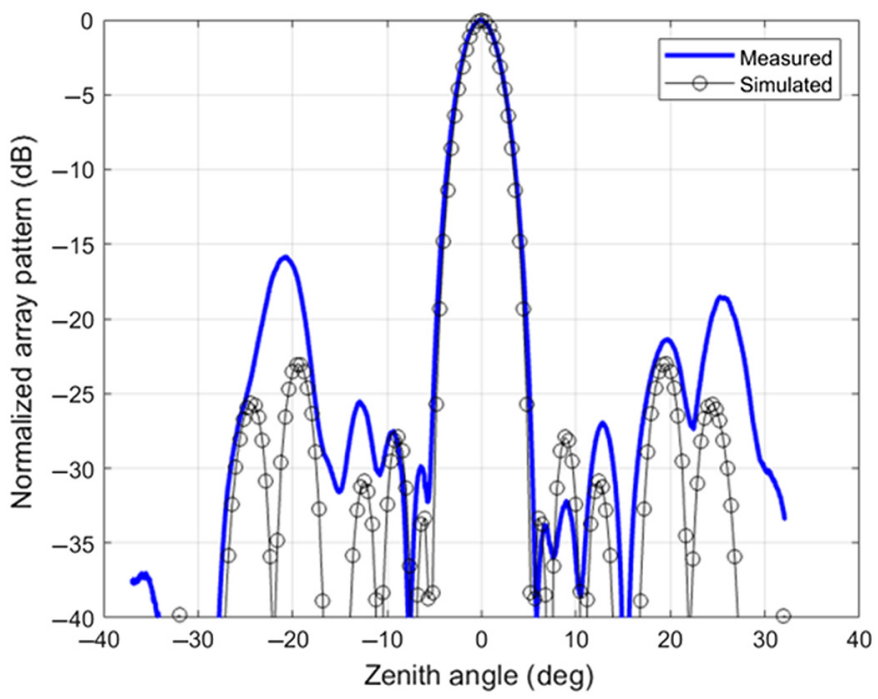

Fig. 8 Far-field pattern (black line) and focused near field (blue line) of tiles 1-24 (full HBA subarray) at $150 \mathrm{MHz}$. 


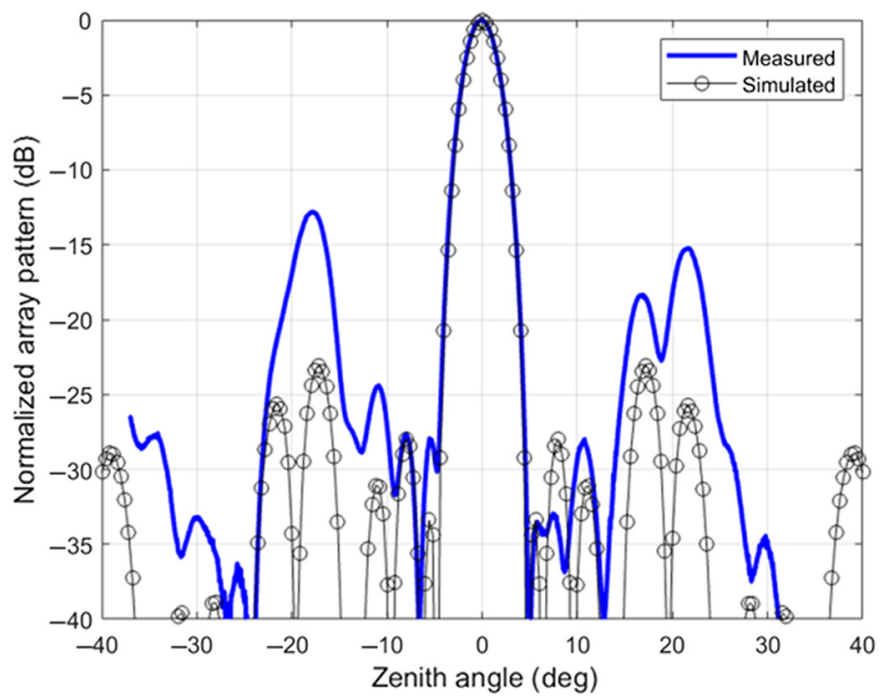

Fig. 9 Far-field pattern (black line) and focused near field (blue line) of tiles 1-24 (full HBA subarray) at $180 \mathrm{MHz}$.

\section{Radial Rasters as an Efficient Scan Strategy}

The results in Sec. 2 concern a single-linear scan performed along the north-west direction. Additional linear scans were performed with an angular offset of 22.5 deg to achieve a more complete coverage of the $u-v$ plane (directional cosines). All angular steps were performed with two orientations of the UAV-mounted dipole, i.e., parallel and orthogonal to the speed vector to sample both the $\theta$ and $\varphi$ components of the radiation patterns. Each raster has been split as two flights for each field component, i.e., a total of four flights. The duration of each flight has been $\sim 10 \mathrm{~min}$. The full flight duration capability of the UAV has not been exploited due to the severe wind condition observed during the campaign. A larger margin on the battery charge has been maintained for safety reasons. Longer flight durations up to $40 \mathrm{~min}$ are now available with modern UAVs operating in calm wind condition. The measured results for the north-west polarized elements of tile number 9 (see Fig. 2) are shown in Fig. 10.

The north-west radiation pattern cut $(+135 \mathrm{deg}$ from $u$ axis) for the (radial) $\theta$ component [Fig. 10(a)] corresponds to the $E$-plane co-polar pattern for the tile elements polarized along the north-west direction (same cut that is discussed in both Sec. 2 and Ref. 23), where both main lobe and sidelobes are visible. The cut oriented north-east [ $\theta$ component, Fig. 10(a)] instead represents a cross-polar $H$-plane pattern, which is quite low in magnitude as expected. The $H$-plane co-polar pattern is visible in Fig. 10(b) where the (azimuthal) $\varphi$ component is shown.

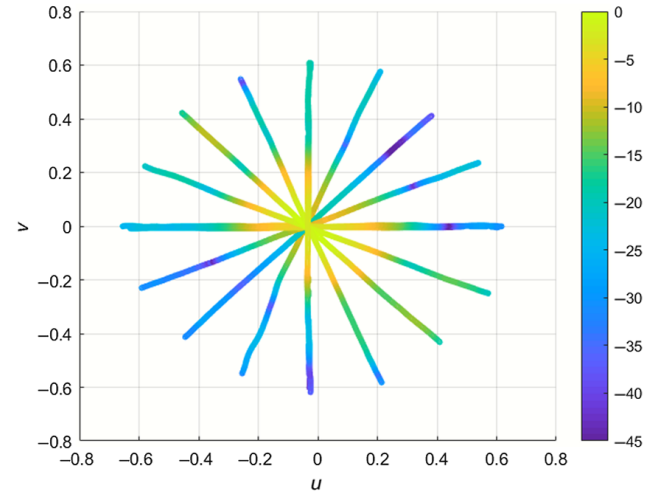

(a)

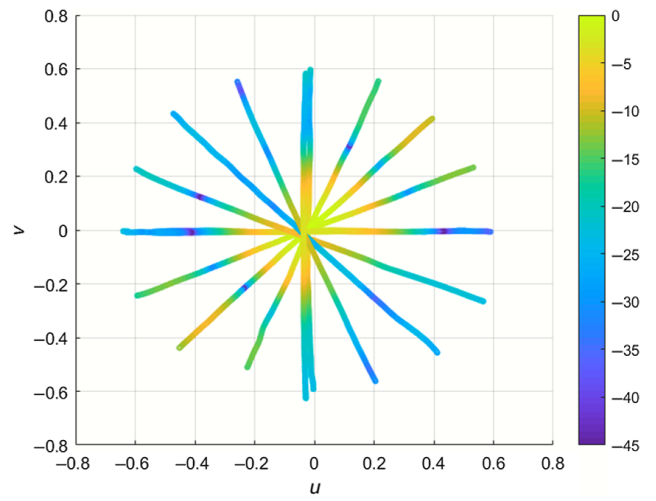

(b)

Fig. 10 Measured (a) $\theta$ component and (b) $\varphi$ component of the radiated pattern of tile 9 at $180 \mathrm{MHz}$ (north-west polarized elements). 


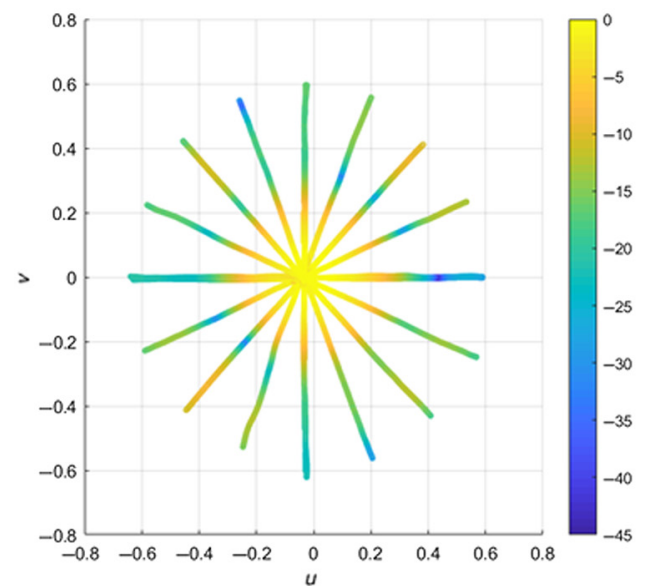

(a)

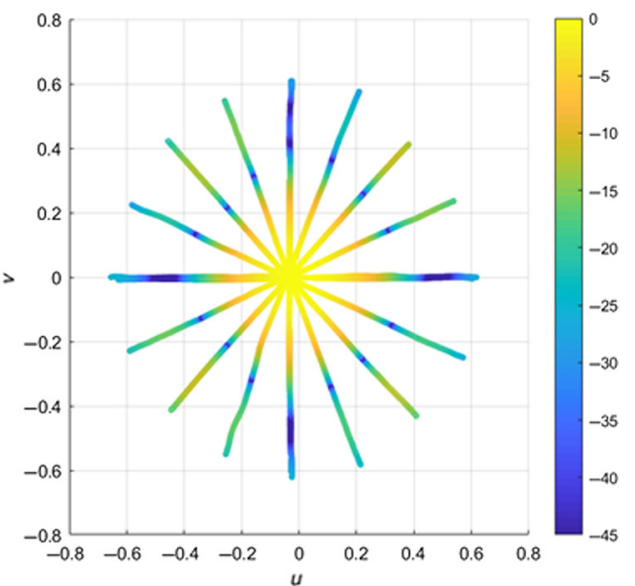

(b)

Fig. 11 (a) Measured and (b) simulated beam patterns for tile 9 at $180 \mathrm{MHz}$ (north-west polarized elements).

The north-east cut shows again both main lobe and first sidelobes. This is consistent with the square geometry of the tiles.

The power pattern, i.e., the combination of the two orthogonal pattern components in Fig. 10, is shown in Fig. 11(a). Both $E$-plane and $H$-plane are now visible on the same plot as north-west and north-east paths, respectively. The symmetry of the beam, which is due to the square geometry of the tiles is clearly visible. The overall pattern is very consistent to the simulated data in Fig. 11(b). The main difference is the depth of the nulls, which is mainly related to source orientation errors, modeling errors, and of course manufacturing and position uncertainties of the real tile. Manufacturing tolerances are neither calibrated nor corrected for in the analog beamformer.

The near-field focusing method described in Sec. 2 has been applied to the full HBA shown in Fig. 2. The result is shown in Fig. 12(a) and is in good agreement with the simulation (b). The beam symmetry and width are consistent. Artifacts are still visible in both the north-west and north-east paths. Nevertheless, these results provide a good in situ validation of the HBA substation.

For brevity, we have only presented results for the HBA elements polarized along the northwest direction. However, similar results have been achieved for the north-east ones.

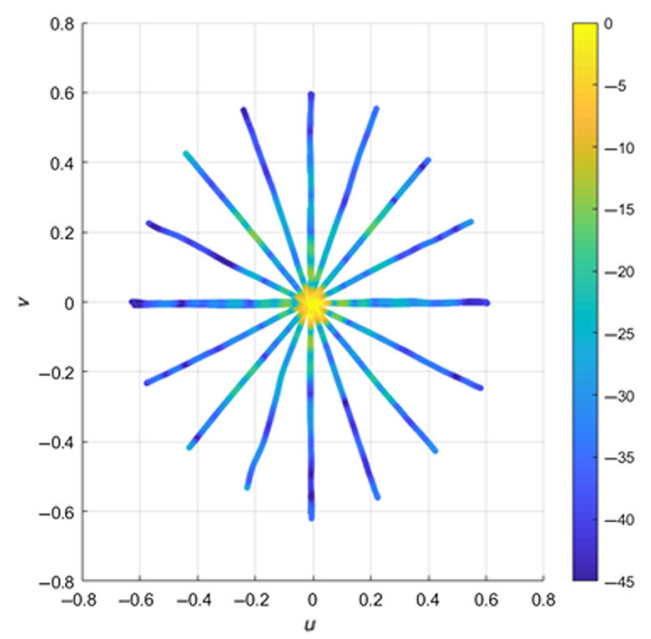

(a)

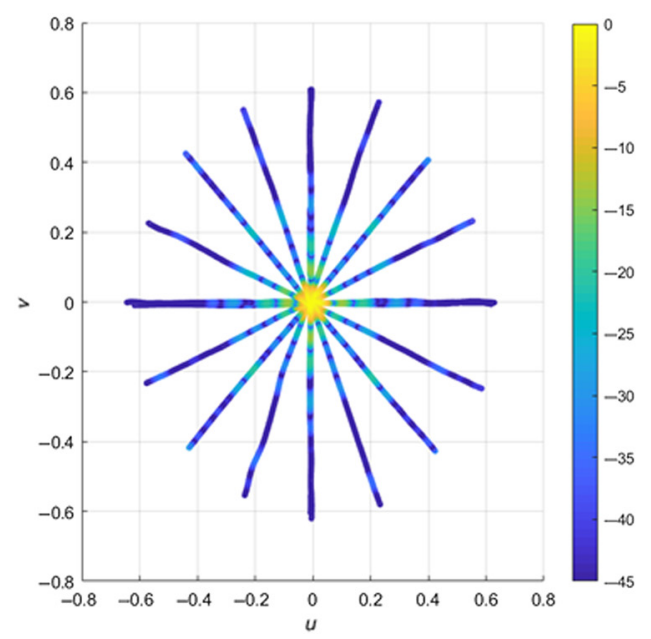

(b)

Fig. 12 (a) Measured and (b) simulated beam patterns of the HBA array in Fig. 2 at $180 \mathrm{MHz}$ (north-west polarized elements). 


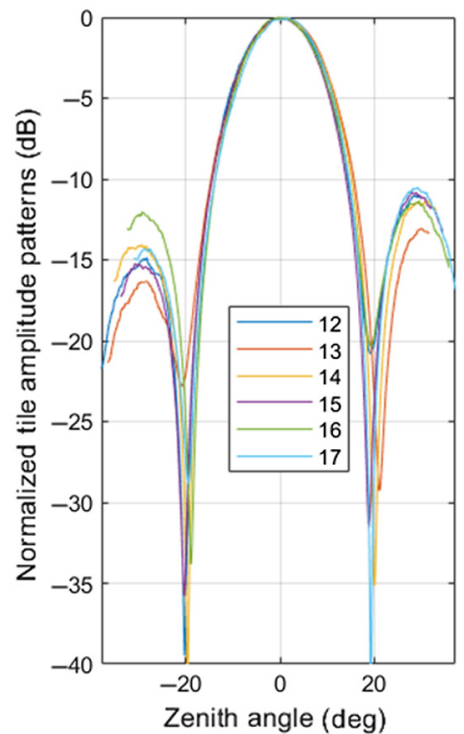

(a)

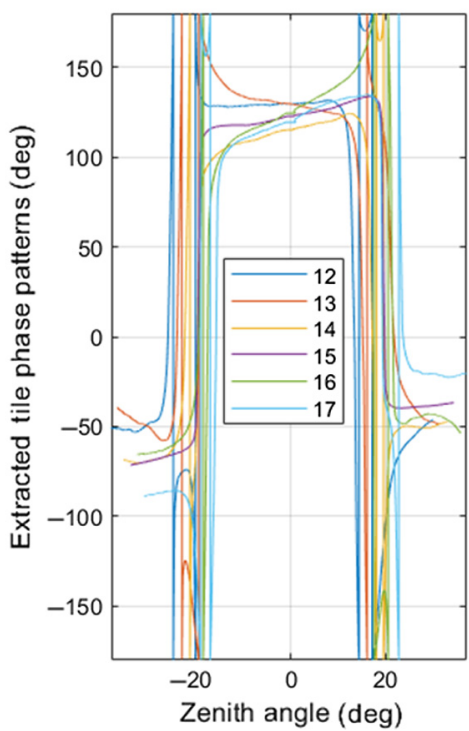

(b)

Fig. 13 E-plane radiation patterns for tiles $12-17$ at $180 \mathrm{MHz}$ : (a) magnitude and (b) phase.

\section{Far-Field Reconstruction}

This section presents an alternative strategy to partially overcome the artifacts due to near-field focusing method already discussed in Sec. 2. It is based on the consideration that the performed UAV flights satisfy the far-field condition for the tiles but not for the array of tiles. For the tiles, both amplitude and phase of the acquired signals are available (complex voltages). The amplitude patterns can be easily obtained by geometrical considerations, i.e., the amplitude data for each tile (after removal of UAV pattern and path loss, see for example Fig. 3) are expressed as a function of a local reference system centered on the tile itself, instead of the center of the full array. The resulting patterns for tiles 12-17 are shown in Fig. 13. The frequency of $180 \mathrm{MHz}$ has been selected for this example because it represents a worst case for the near-field focusing artifacts.

As far as far-field phase patterns are concerned, their determination is less straightforward because the UAV-mounted RF source is not phase-locked to the on-ground acquisition system of the telescope. In other words, the relative phase between transmitter and receivers is drifting during the flight in an uncontrolled way. For this reason, only differential phase data can be used. ${ }^{24}$ In Ref. 25, a reference antenna with known phase pattern was placed in the proximity of the SKA-LOW array prototype to reconstruct a near-field phase pattern. In this campaign, no reference antenna was available. Therefore, one of the central tiles (i.e., tile number 15) is used as reference. This, of course, relies on the knowledge of its phase pattern by simulations [see Fig. 13(b), violet curve]. Under this hypothesis, the phase patterns of all other tiles $\varnothing_{j}$ can be computed as

$$
\varnothing_{j}=\varnothing_{i}+\varphi_{j}-\varphi_{i}+k\left(r_{j}-r_{i}\right),
$$

where the time/position dependence of all the terms has been understood, $\varnothing_{i}$ is the phase pattern of the reference tile ( $i=15$ in our case), $\varphi_{j}$ and $\varphi_{i}$ are the phase of the acquired complex voltages, $k$ is the wave number, and $r_{j}, r_{i}$ are the distances between the UAV-mounted source and the centers of tiles $j$ and $i$, respectively. It should be mentioned that in Eq. (1), the direction dependence of the source pattern has been neglected (it is in the order of a few degrees). Moreover, only the co-polar component is considered (transmitter and receiver are matched in polarization). The relative distances $r_{j}$ and $r_{i}$ are computed exploiting the UAV position data measured by GNSS and the knowledge of the tile center positions. (Nominal data have been used, however, an accurate measurement with ground-based instruments such as total station or GNSS is viable.) The resulting reconstructed far-field phase patterns for tiles 12-14 and 16-17 are shown in Fig. 13(b). 


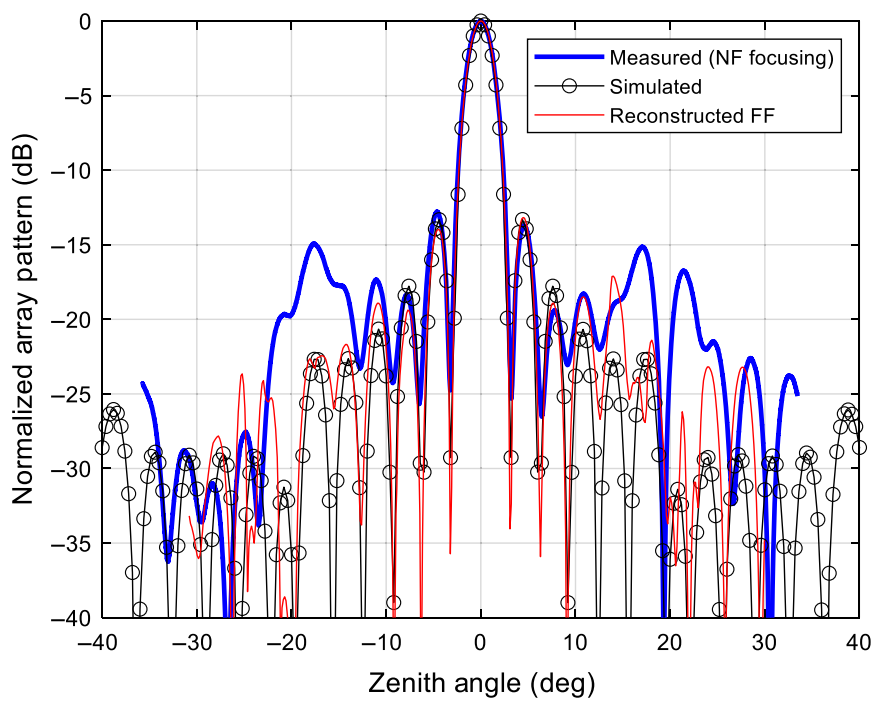

Fig. 14 E-plane radiation pattern for tiles $12-17$ at $180 \mathrm{MHz}$.

They are quite similar to each other because local reference systems (centered on each tile) have been adopted. Each of them shows narrow anomalies where the phase rotates of 360 deg around the zenith angle $\pm 20 \mathrm{deg}$. These anomalies are due to the two nulls on the pattern of the reference tile. At the nulls, the phase exhibits abrupt variations of $180 \mathrm{deg}$. In these regions, the accuracy of the models is generally lower and therefore, a perfect cancellation between the terms $\varnothing_{i}$ and $\varphi_{i}$ does not occur in Eq. (1). A reference antenna with a smoother behavior would be desirable to avoid such anomalies (this can be implemented by either activating only one dipole within a tile that will be hence used as reference only or exploiting an additional external reference antenna in the proximity of the HBA). Nevertheless, after summation of the tile patterns in Fig. 13, the resulting beam in Fig. 14 (red curve) is more consistent to the far-field simulation (black curve) than the previous near-field focusing data (blue curve). It should be mentioned that, before summation, the tile phase patterns in Fig. 13 have been both converted to the same reference system by exploiting again the knowledge of the tile center positions and equalized at zenith (array calibration). The angular regions where the discrepancies occur are consistent with the position of the reconstructed far-field phase pattern anomalies in Fig. 13. A better agreement has been also achieved at $124 \mathrm{MHz}$ (see Fig. 15) using the same reconstruction method.

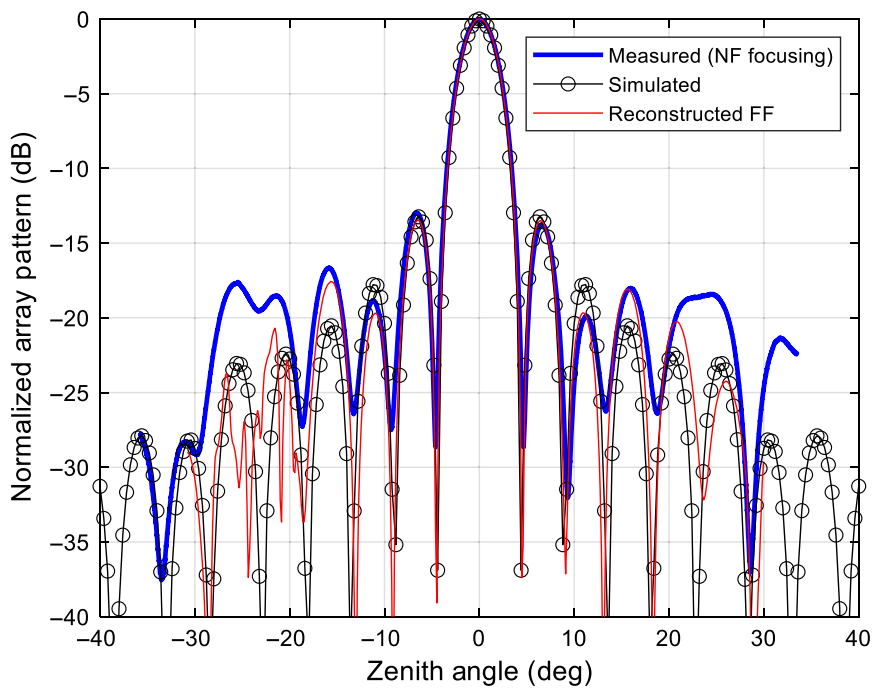

Fig. 15 E-plane radiation pattern for tiles $12-17$ at $124 \mathrm{MHz}$. 
The overall improvement has been quantified computing the average (along zenith angle) of the weighted logarithmic difference $\Delta_{w, \log }$ in Ref. 26 with $\beta=0.5$ between measurements and simulation. For the near-field focusing (blue curve), the quantity $\Delta_{w, \log }$ is 0.89 and $1.15 \mathrm{~dB}$ at 124 and $180 \mathrm{MHz}$, respectively. A smaller $\Delta_{w, \log }$ of $0.68 \mathrm{~dB}$ has been achieved for the reconstructed far-field case (red curve) at both frequencies.

The method presented in this section has only been applied to the linear array of tiles 12-17, which is close to the north-west scan path performed by the UAV (see Fig. 2). The array of tiles 6-11 has not been considered because of the faulty tile. The best condition for the presented farfield reconstruction is a scan path that intersects the maxima of the tile beams, whose pointing is set before the flight. Otherwise, the information of the principal cut of the tile radiation pattern is not available. A linear flight parallel to the array lattice direction (almost north-west in this case) will intersect the maximum number of tile beam maxima. According to these considerations, a Cartesian raster with $6 \times 6$ orthogonal linear scans and a spacing of $5.15 \mathrm{~m}$ (distance between tile centers) would have allowed the measurements of all the tile patterns in their principal planes and the subsequent reconstruction of the full array far-field pattern along the north-west and northeast planes using the method presented in this section. It should be noted that a raster that is suitable for a near-field to far-field transformation would have required a spacing of $<\lambda / 2$, i.e., $0.8 \mathrm{~m}$ at $180 \mathrm{MHz}$, with a strong impact on the UAV flight time requirement. Moreover, a computationally heavy inversion algorithm must be applied ${ }^{27}$ to transform the scan paths performed by UAV in the near field. The presented solution, together with the exploitation of a smoother and well-known reference antenna could be considered for the characterization of the regular arrays of SKA-MID.

\section{Conclusion and Future Developments}

The application of near-field focusing to an array of LOFAR-HBA tiles pointed in the same farfield direction demonstrated that a useful end-to-end system validation can be performed even considering only a limited angular range around the beam axis. Simulated and measured results are in good agreement as far as beamwidth and first sidelobes are concerned. The method also pointed out the presence of a faulty tile in the Eastern HBA subarray of the CS302 station.

The combination of a few linear scans with different orientations has been performed to achieve a radial raster. This procedure confirmed the agreement between measurements and simulations on the full $u-v$ plane, although with limited coverage. The angular step of $22.5 \mathrm{deg}$ could be reduced at the expense of a longer flight/scan duration.

A far-field reconstruction method has been proposed and validated on a linear subarray of the HBA substation. It allows one to partially overcome the artifacts of the near-field focusing strategy exploiting the usage of a known reference antenna and the knowledge of the tile positions. This suggests a validation procedure for regular aperture arrays such as SKA-MID that is based on a Cartesian raster in the near field with a spacing that is equal to the tile spacing, which is several times larger than $\lambda / 2$. This efficient scan strategy will provide far-field pattern information on the principal planes only, which could already be satisfactory as far as validating stations in situ is concerned.

\section{Acknowledgments}

The authors would like to acknowledge M. J. Norden from ASTRON, The Netherlands, and A. Lingua, P. Maschio, and I. Aicardi from Politecnico di Torino, Italy for their valuable technical support. The authors also would like to thank Dion Kant and Michel Arts for the WIPL-D simulations. This work was supported by the Netherlands Organization for Scientific Research.

\section{References}

1. M. P. van Haarlem et al, "LOFAR: the low-frequency array," Astron. Astrophys. 556, A2 (2013). 
2. A. J. J. van Es et al., "A prototype model for evaluating SKA-LOW station calibration," Proc. SPIE 11445, 1144589 (2020).

3. P. Bolli et al., "Test-driven design of an active dual-polarized log-periodic antenna for the square kilometre array," IEEE Open Journal of Antennas and Propagation 1, 253-263 (2020).

4. I. Farhat et al., "Characterization of 36 meter square mid-frequency radio astronomy prototype antenna array," in IEEE Conf. Antenna Meas. and Appl., Vasteras, pp. 1-3 (2018).

5. Y. Zhang et al., "On the front-end design of mid-frequency aperture array for square kilometre array," Exp. Astron. 46, 357-380 (2018).

6. G. W. Kant et al., "EMBRACE: a multi-beam 20,000-element radio astronomical phased array antenna demonstrator," IEEE Trans. Antennas and Prop. 59(6), 1990-2003 (2011).

7. U. Kiefner et al., "Holographic calibration of phased array telescopes," Radio Sci. 56, e2020RS007171 (2021).

8. P. Salas et al., "Tied-array holography with LOFAR," Astron. Astrophys. 635, A207 (2020).

9. S. J. Wijnholds and W. A. van Cappellen, "In situ antenna performance evaluation of the LOFAR phased array radio telescope," IEEE Trans. Antennas Propagat. 59(6), 1981-1989 (2011).

10. A. Nelles et al., "Calibrating the absolute amplitude scale for air showers measured at LOFAR," J. Instrum. 10(11), P11005 (2015).

11. E. de Lera Acedo et al., "SKA aperture array verification system: electromagnetic modeling and beam pattern measurements using a micro UAV," Exp. Astron. 45(1), 1-20 (2018).

12. P. Bolli et al., "Antenna pattern characterization of the low-frequency receptor of LOFAR by means of an UAV-mounted artificial test source," Proc. SPIE 9906, 99063V (2016).

13. G. Pupillo et al., "Medicina array demonstrator: calibration and radiation pattern characterization using a UAV-mounted radio-frequency source," Exp. Astron. 39(2), 405-421 (2015).

14. P. Bolli et al., "From MAD to SAD: the Italian experience for the low frequency aperture array of SKA1-LOW," Radio Sci. 51(3), 160-175 (2016).

15. G. Virone et al., "Strong mutual coupling effects on LOFAR: modeling and in situ validation," IEEE Trans. Antennas Propag. 66(5), 2581-2588 (2018).

16. F. Paonessa et al., "UAV-based pattern measurement of the SKALA," in IEEE Int. Symp. Antennas and Propag./USNC/URSI Natl. N. Am. Radio Sci. Meeting Location, Vancouver, Canada (2015).

17. P. Bolli et al., "Near-field experimental verification of the EM models for the LOFAR radio telescope," IEEE Antennas Wirel. Propag. Lett. 17(4), 613-616 (2018).

18. P. Di Ninni et al., "Electromagnetic analysis and experimental validation of the LOFAR radiation patterns," Int. J. Antennas Propag. 2019, 1-12 (2019).

19. F. Paonessa et al., "SKA-low prototypes deployed in Australia: synoptic of the UAV-based experimental results," Radio Sci. Lett. 2, 1-5 (2020).

20. J. Sherman, "Properties of focused apertures in the Fresnel region," IRE Trans. Anntenas Propag. 10(4), 399-408 (1962).

21. P. Nepa and A. Buffi, "Near-field-focused microwave antennas: near-field shaping and implementation," IEEE Antennas Propag. Mag. 59(3), 42-53 (2017).

22. F. Paonessa et al., "UAV-based antenna measurements: improvement of the test source frequency behavior," in IEEE Conf. Antenna Meas. and Appl., Västerås, Sweden (2018).

23. G. Virone et al., "Preliminary results on the verification of the LOFAR-HBA with a flying test source," in 15th Eur. Conf. Antennas and Propag., pp. 1-4 (2021).

24. F. Paonessa et al., "Differential phase patterns of the LOFAR LBA array measured in situ," in 12th Eur. Conf. Antennas and Propag., London (2018).

25. L. Ciorba et al., "Near-field phase reconstruction for UAV-based antenna measurements," in 13th Eur. Conf. Antennas and Propag., pp. 1-4 (2019).

26. S. Pivnenko et al., "Comparison of antenna measurement facilities with the DTU-ESA $12 \mathrm{GHz}$ validation standard antenna within the EU antenna centre of excellence," IEEE Trans. Antennas Propag. 57(7), 1863-1878 (2009).

27. J. L. A. Quijano and G. Vecchi, "Field and source equivalence in source reconstruction on 3D surfaces," Prog. Electromagn. Res. 103, 67-100 (2010). 
Giuseppe Virone received his degree in electronic engineering (summa cum laude) and his $\mathrm{PhD}$ in electronics and communication engineering from the Politecnico di Torino, Turin, Italy, in November 2001 and 2006, respectively. He is currently a senior researcher at the Istituto di Elettronica e di Ingegneria Informatica e delle Telecomunicazioni (IEIIT) of Italian National Research Council (CNR). He joined IEIIT as a research assistant in 2002. He coordinated more than 15 scientific projects funded by both the industry and other scientific research organizations and joined more than 30 research projects as a collaborator. He authored 43 journal papers, 134 conference papers, and 3 European patents. His activities concern the design, numerical analysis, and characterization of microwave and millimeter waveguide passive components for feed systems, antenna arrays, frequency selective surfaces, compensated dielectric radomes, and industrial sensing applications.

Fabio Paonessa received his BS and his MS degrees in biomedical engineering and his $\mathrm{PhD}$ in electronics engineering from the Polytechnic University of Turin in 2008, 2010, and 2017, respectively. From 2011 to 2012, he was a research assistant in the Department of Electronics at the Polytechnic University of Turin. In 2013, he joined the Applied Electromagnetics Group of IEIIT at CNR. He became a researcher in 2018. His activities include the scientific applications of the unmanned aerial vehicles for the characterization of antenna arrays and radar systems and wireless sensor networks related applications.

Lorenzo Ciorba received his master's degree (110/110) in mathematical engineering from the Politecnico di Torino, Turin, Italy, in March 2018, with the thesis "Hybrid Antenna Measurement and Simulations" with Prof. G. Vecchi as a supervisor. In June 2018, he joined as a research fellow in the Applied Electromagnetics and Electronic Devices Group of the IEIIT at CNR. From November 2018, he has been a PhD student in electrical, electronics, and communications engineering at the Politecnico di Torino. His scientific interests regard computational electromagnetics and characterization of antennas, in particular UAV-based near field antenna measurements.

Stefania Matteoli received her BS and MS (cum laude) degrees in telecommunications engineering and her $\mathrm{PhD}$ in remote sensing from the University of Pisa, Pisa, Italy, in 2003, 2006, and 2010, respectively. She is currently a permanent researcher at the National Research Council of Italy of the Institute of Electronics, Computers, and Telecommunication Engineering. From January 2010 to December 2016, she was first a postdoctoral fellow and then a temporary researcher in the Department of Information Engineering, University of Pisa, Pisa, Italy. From May 2008 to October 2008, she was a visiting student at Chester F. Carlson Center for Imaging Science, Rochester Institute of Technology, Rochester, New York, USA. She is an associate editor of the IEEE Geoscience and Remote Sensing Letters and the SPIE Journal of Applied Remote Sensing. Her main research interests include signal and image processing applied to various remote sensing applications and to antenna array data processing.

Pietro Bolli received his Laurea degree in electronic engineering and his $\mathrm{PhD}$ in computer science and telecommunications engineering from the University of Florence, Florence, Italy, in 1999 and 2003, respectively. In 2002, he started his professional career as a microwave engineer at the Italian National Institute for Astrophysics (INAF) conducting research in the field of technology applied to radio astronomy. He is currently senior technologist at the INAF Arcetri Astrophysical Observatory, where he is involved in the design, characterization, and calibration of the low-frequency instrument of the square kilometer array. He is the Italian responsible for the protection of the frequency bands used by radio astronomers and represents INAF in the Committee on Radio Astronomy Frequencies. He is also officer of the Commission $\mathbf{J}$ (Radio Astronomy) of the Italian Committee of the Union Radio Scientifique Internationale. $\mathrm{He}$ is a co-author of about 140 scientific publications, which have appeared in international referred journals and conferences.

Stefan J. Wijnholds received his MSc degree in astronomy and his MEng degree in applied physics (both cum laude) from the University of Groningen, Groningen, The Netherlands, in 2003 and his PhD (cum laude) from Delft University of Technology, Delft, The Netherlands, 
in 2010. After his graduation in 2003, he joined the R\&D Department of ASTRON, the Netherlands Institute for Radio Astronomy, Dwingeloo, The Netherlands, where he works on the development of the next generation of radio telescopes based on phased array technology. From 2006 to 2010, he was also affiliated with the Delft University of Technology, Delft, The Netherlands. Since 2016, he has been affiliated in the Electrical and Electronic Engineering Department, University of Stellenbosch, Stellenbosch, South Africa, as an extraordinary (associate) professor. In 2018, he became a senior researcher at ASTRON overseeing work on the institutional technology development roadmap to address the big data challenges posed by large phased array systems. His research interests lie in the area of array signal processing, specifically calibration and imaging, and system design of the next generation of radio telescopes.

Giuseppe Addamo received his $\mathrm{PhD}$ in electronic and communication engineering from the Politecnico di Torino, Turin, Italy, in 2007. Since 2012, he has been a researcher with the Instituto di Elettronica e di Ingegneria dell'Informazione e delle Telecomunicazioni, Consiglio Nazionale delle Ricerche, Turin, Italy. His research interests are in the areas of microwave leaky antennas, dielectric radomes, high-power feed systems (e.g., orthomode transducers, microwave filters), corrugated horns, frequency-selective surfaces, and large dielectric radomes. 